\title{
Analysis of the partially premixed combustion in a lab- scale swirl-stabilized burner fueled by a methane-hydrogen mixture
}

\author{
Michele Stefanizzi ${ }^{1}$, Saverio Stefanizzi ${ }^{1}$, Vito Ceglie ${ }^{1, *}$, Tommaso Capurso ${ }^{1}$, Marco Torresi ${ }^{1}$, \\ and Sergio Mario Camporeale ${ }^{1}$ \\ ${ }^{1}$ Department of Mechanics, Mathematics and Management - Polytechnic University of Bari, Via Re \\ David, 200, 70125 Bari, Italy
}

\begin{abstract}
Nowadays hydrogen is gaining more and more attention by Industry, Academia and Politics. Being a carbon free fuel, it is supposed to have a key role in the future energy scenario, especially if produced by renewable sources. The use of mixtures of hydrogen and conventional hydrocarbons in gas turbines is one of the most promising technical solutions for obtaining a sustainable combustion during the transition toward a full decarbonization. For this reason, it is fundamental to investigate the behaviour of fuels enriched with hydrogen in combustion processes. In this work, a lab-scale swirled premixed burner has been investigated by means of a fully 3D URANS approach. Firstly, a numerical simulation with cold flow has been performed to validate the model against experimental data. Then, reactive flow simulations have been performed. Initially, a combustion with $100 \%$ methane was considered. Then, a $30 \%$ by volume hydrogen blending has been investigated. The partially premixed combustion model has been implemented to take into account the inhomogeneities of the mixture at the chamber inlet. The variation of the flame structure due to the hydrogen enrichment will be described in terms of the temperature and species concentration distributions.
\end{abstract}

\section{Introduction}

Nowadays the effort by Industry, Academia and Politics towards a global decarbonization process is becoming more and more essential. In this scenario, there is a great interest toward the use of hydrogen, being a carbon free fuel [1-4]. In fact, the only sustainable hydrogen is known as "green hydrogen", which is obtained by the electrolysis of water in special electrochemical cells powered by electricity produced from renewable sources. It can play a key role in the future energy scenario, being a means of chemically storing excess renewable energy (the so-called power to gas technology, P2G) produced in low demand hours [5]. The use of mixtures of hydrogen and conventional hydrocarbons in gas turbines is one of the most promising technical solutions for obtaining a sustainable combustion during the transition period toward a full decarbonization. Its wide flammability limits can allow lean operation of gas turbine combustors, thus reducing the $N O_{x}$ emissions [6]. Nevertheless, there are still several challenging technical issues that must be addressed such as the potential flashback

\footnotetext{
*e-mail: vito.ceglie@ poliba.it
} 
and autoignition due to the significantly higher flame speeds and shorter autoignition times [7-9]. Several research groups are working both on experimental and numerical activities concerning the premixed combustion of natural gas enriched with hydrogen [10]. Furthermore, an excessive lean combustion can cause the occurrence of dangerous thermoacoustic instabilities [11-15]. Usually, in the numerical approach, the mixture of fuel and oxidizer is assumed perfectly premixed. However, this is an idealization, which often goes in contrast with the reality; hence, in this work the authors take into account the real mixture formation by simulating both the air inlet and the fuel injection.

In the present work, the main advantages and disadvantages related to combustion with hydrogen are highlighted by carrying out a thermo-fluid dynamic study on a swirl-stabilized lean-premixed lab-scale burner. This burner, both in the case of cold and hot flow, has been simulated by means of the commercial software ANSYS Fluent ${ }^{\circledR}$. In the case of cold flow, two cases have been considered: in the first, only the flow of combustion air is simulated and an analysis of the flow fields and the characteristic period of the Precessing Vortex Core (PVC) is carried out; in the second case the fuel is introduced in order to investigate the mixing processes between the comburent and the fuel. For this last analysis, two types of fuel composition are analyzed: injection of methane only $\left(\mathrm{CH}_{4}\right)$ and injection of a mixture of methane enriched with hydrogen $\left(\mathrm{H}_{2}\right)$ at $30 \% \mathrm{v}$ (on volume basis). Actually, it is believed that $30 \% \mathrm{v}$ hydrogen in hydrogen-natural gas mixtures can represent a limit to the correct functioning of modern thermo-electric plants without the need to make changes to the gas turbines [16]. In the numerical analysis of the reacting system with both the combustion of methane and of the methane-hydrogen mixture, temperature and species concentration distributions inside the combustion chamber are considered.

\section{Case Study}

In this work a lab-scale swirled premixed burner of the Louisiana State University has been studied, whose geometry available in the literature has been retrieved by [17-20]. As depicted in Figure 1, the burner consists of a $45^{\circ}$ swirl vane with 8 straight blades fitted with a cylindrical center body. The annulus is not characterized by a constant cross section. Indeed, its inner diameter decreases from $19.05 \mathrm{~mm}$ (at the air inlet section) to $12.7 \mathrm{~mm}$ (at a distance of $76.2 \mathrm{~mm}$ from the dump plane). Moreover, $D=12.7 \mathrm{~mm}$ is assumed as the burner's characteristic dimension. Hence, the swirler is located at a distance of $14 D$ from the dump plane, and it is characterized by a geometric Swirl Number $S_{g}=0.82$, as defined by equation 1 :

$$
S_{g}=\frac{2}{3}\left(\frac{1-\left(d_{h} / d\right)^{3}}{1-\left(d_{h} / d\right)^{2}}\right) \tan \theta
$$

where $d_{h}=20.96 \mathrm{~mm}$ is the swirler inner diameter, $d=34.93 \mathrm{~mm}$ is the outer diameter of the air delivery pipe and $\theta=45 \mathrm{deg}$ is the swirl angle. Furthermore, the swirl vane has an axial dimension of $12.70 \mathrm{~mm}$ and blade height, and thickness equal to $5.08 \mathrm{~mm}$ and $2.38 \mathrm{~mm}$, respectively. The fuel mixture is injected through 8 equally spaced holes, with a diameter of $0.7874 \mathrm{~mm}$, located downstream of the backward facing step due to the cross section restriction of the center body, exactly $9.53 \mathrm{~mm}$ from it (see Figure 1). Moreover, the fuel injection holes are inclined $11.46 \mathrm{deg}$ with respect to the radial direction.

\section{Numerical Setup}

The numerical investigation has been carried out by means of ANSYS Fluent ${ }^{\circledR}$ v17.2 with a fully 3D URANS approach. For the non-reacting flow (only air), the Reynolds Stress Model 


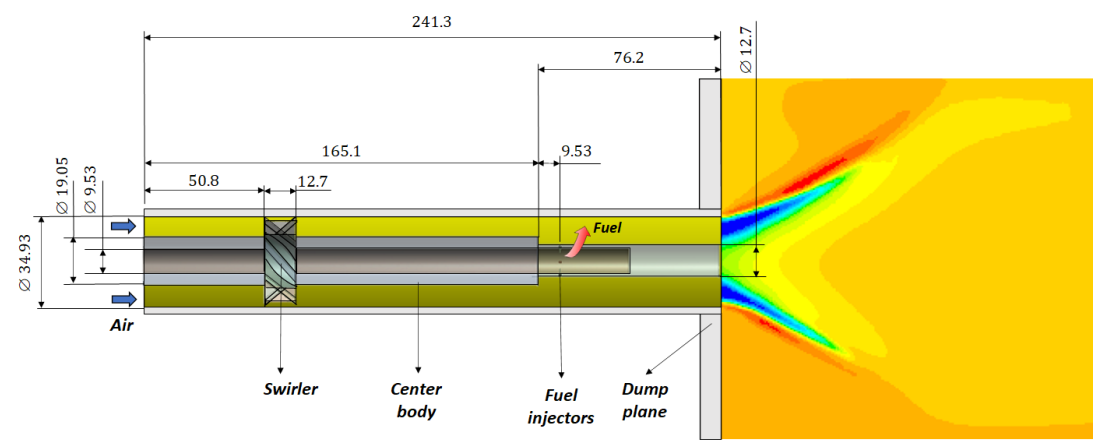

Figure 1. View of the investigated burner with its main parts and dimensions (in $\mathrm{mm}$ ).

(RSM) has been used as turbulence model and the hypothesis of incompressible and adiabatic flow has been assumed considering atmospheric operating conditions $\left(T_{a m b}=298.15 \mathrm{~K}\right.$ and $p_{a m b}=101325 \mathrm{~Pa}$ ). Among turbulence models provided in ANSYS Fluent ${ }^{\circledR}$, the RSM has been used since it has showed the best results in terms of velocity profiles with respect to the experimental ones. This can be explained since the RSM takes into account the anisotropy of the turbulence, which allows a good simulation of strongly swirled flows (typical in our case study). Moreover, a pressure-based solver with a SIMPLE pressure-velocity coupling scheme has been used. Finally, a time step size $\Delta t=10^{-2} \mathrm{~s}$ has been defined in order to take into account the non-stationary phenomena related to the Precessing Vortex Core (PVC). Indeed, as evidenced by experimental activities in $[18,21]$, the PVC for the non-reacting flow is characterized by a frequency equal to $3 \mathrm{~Hz}$.

For the reacting flow, a Partially Premixed Combustion Model is used to simulate the combustion process, since there is neither a perfectly premixed nor a diffusive flame. Furthermore, the Steady Diffusion Flamelet approach is selected in order to consider all the reaction phenomena released from the chemical equilibrium hypothesis because of the flame stretching due to the interaction with the external turbulent velocity field. In addition to the default Fluent library for the thermodynamic properties, the GRI-mech 2.11 [22] has been used as Gas-Phase CHEMKIN Mechanism File in order to generate the PDF-table. It consists of 49 chemical species and 277 reactions. The Discrete Ordinates Model (DO) has been chosen as radiation model. In this case, a COUPLED solution scheme has been used because it is better than segregated algorithms in simulating compressibility effects, which surely are noteworthy in the combustion. For the hot cases simulations, the time step size is halved respect to the non-reacting flow.

\subsection{Computational Domain and Boundary Conditions}

Figure 2 shows the computational domain used in this work. The combustion chamber is cylindrical with the axial and the radial dimensions equal to $20 D$ and $12 D$, respectively. Three different unstructured grids have been generated with different levels of refinement. Figure 3 shows the comparison between numerical and experimental mean axial velocity profiles normalized with respect to the air bulk velocity, $u / u_{0}$. The profiles have been computed in the case of non-reacting flow at different axial locations downstream of the dump plane. In the end, grid number 2 has been chosen as a good compromise between computational costs and results accuracy. In details, the selected mesh has been generated via ANSYS Workbench with a number of elements close to $3.7 \mathrm{M}$. A uniform mesh sizing of $1.5 \mathrm{~mm}$ has been chosen from the air inlet to a chamber section located at $60 \mathrm{~mm}$ downstream the dump plane; then, 


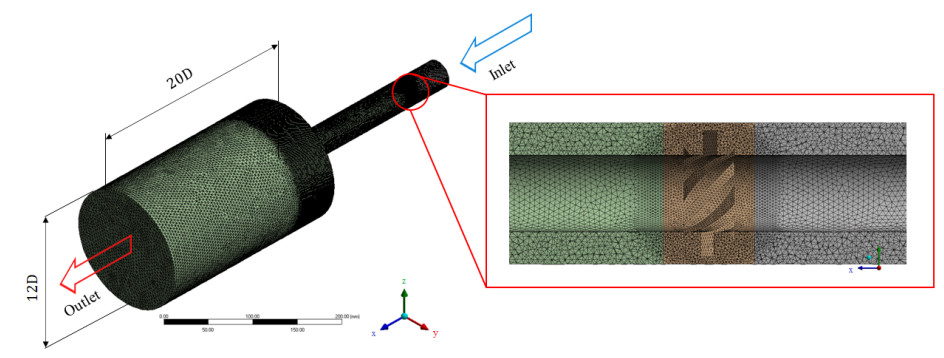

Figure 2. View of the numerical domain with a detail of the mesh sizing for the swirler vane.

with a growth rate of 1.2, the cell size gradually increases up to $4 \mathrm{~mm}$ towards the outlet section, certainly with lesser computational interest. In addition, the domain containing the swirler has been refined with a finer sizing by decreasing the cells dimension from $1.5 \mathrm{~mm}$ to $0.75 \mathrm{~mm}$ with a growth rate of 1.2 (Figure 2). Finally, to allow an accurate fuel flow rate injection, for the reacting flow simulations, the fuel injectors have been discretized with a local face sizing of $0.15 \mathrm{~mm}$.

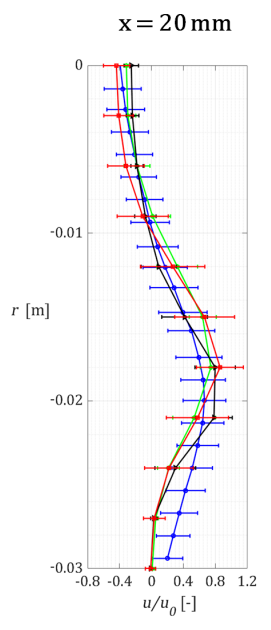

(a)

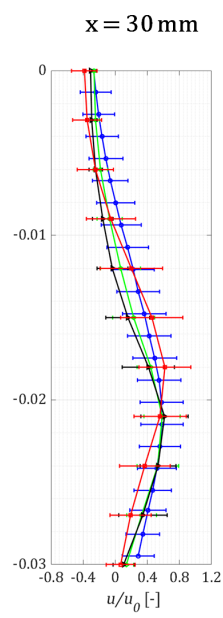

(b)

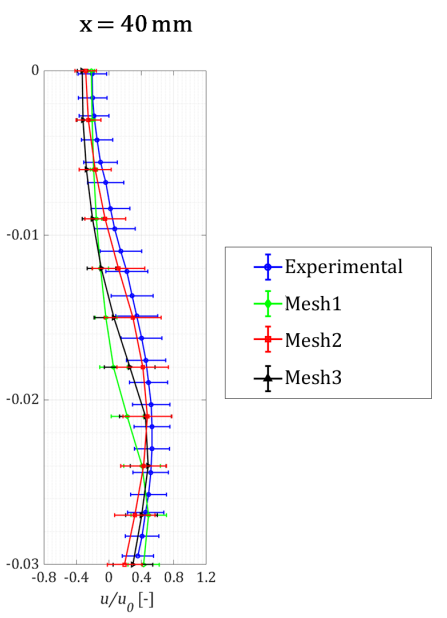

(c)

Figure 3. Comparison for different meshes between numerical and experimental normalized mean axial velocity profiles, $u / u_{0}$, for non-reacting flow, at different axial locations $(x=$ $20 \mathrm{~mm}$ (a), $x=30 \mathrm{~mm}$ (b), $x=40 \mathrm{~mm}$ (c)).

\subsubsection{Boundary Conditions - Non-reacting flow}

For the air inlet section, a velocity-inlet boundary condition has been chosen, setting a velocity magnitude of $11.83 \mathrm{~m} / \mathrm{s}$ (i.e., the air bulk velocity, $u_{0}$ ) and a turbulence intensity of $5 \%$. An outflow condition has been applied to the domain outlet. This type of boundary condition is used to model flow exits where the details of the flow velocity and pressure are not known prior to solve the flow problem. Moreover, the chamber surface is treated with a slip wall type boundary condition, whereas all the other walls of the computational domain are characterized by a wall no-slip condition. 


\subsubsection{Boundary Conditions - Reacting flow}

Referring to the reacting flow simulations, the inlet-velocity boundary conditions for the fuel injectors have been introduced considering two cases: injection of pure methane $\left(100 \% \mathrm{CH}_{4}\right)$ and injection of a mixture of methane enriched with $30 \% \mathrm{v}$ of hydrogen $(70 \% \mathrm{v} \mathrm{CH}+30 \% \mathrm{v}$ $\mathrm{H}_{2}$ ). Table 1 compares the main parameters for the fuel mixtures of the two aforementioned cases. The inlet radial, $U_{\text {rad, fuel }}$, and tangential, $U_{\text {tan, fuel }}$, velocity components of the fuel mixture have been calculated by assuming constant the inlet air mass flow rate, $\dot{m}_{\text {air }}=9.307 \mathrm{~g} / \mathrm{s}$ and the mean value of the equivalence ratio, $\phi=0.7$, defined as the ratio between the stoichiometric, $\alpha_{s t}$, and actual, $\alpha$, air-fuel ratio. Moreover, it can be noted that $30 \% v$ of hydrogen corresponds to only $5 \% \mathrm{~m}$ in the same mixture. Moreover, in order to consider the effect of the irradiation, the walls internal emissivity has been set to 0.6 and zero heat flux hypothesis has been adopted to simulate an adiabatic system. Furthermore, the fuel is assumed to be injected counter-rotating with respect to the main swirled air flow.

Table 1: Comparison of the main fuel parameters for pure methane and the case of methane enriched with hydrogen.

\begin{tabular}{|l|c|c|}
\hline Parameter & $100 \% \mathrm{CH}_{4}$ & $70 \% \mathrm{CH}_{4}+30 \% v \mathrm{H}_{2}$ \\
\hline$\phi[-]$ & 0.7 & 0.7 \\
$\alpha_{\text {st }}[-]$ & 17.225 & 18.032 \\
$M W[\mathrm{~kg} / \mathrm{kmol}]$ & 16.0 & 11.8 \\
$\alpha[-]$ & 24.65 & 25.76 \\
$\dot{m}_{\text {air }}[\mathrm{g} / \mathrm{s}]$ & 9.307 & 9.307 \\
$\dot{m}_{\text {fuel }}[\mathrm{g} / \mathrm{s}]$ & 0.377 & 0.361 \\
$\rho_{\text {fuel }}\left[\mathrm{kg} / \mathrm{m}^{3}\right]$ & 0.6551 & 0.4796 \\
$U_{\text {rad }, \text { fuel }}[\mathrm{m} / \mathrm{s}]$ & 150.9 & 197.3 \\
$U_{\text {tan,fuel }}[\mathrm{m} / \mathrm{s}]$ & 30.6 & 40.0 \\
$L H V[\mathrm{MJ} / \mathrm{kg}]$ & 50.0 & 53.6 \\
\hline
\end{tabular}

\section{Results and discussion}

\subsection{Non-Reacting Flow}

Figure 4 and Figure 5 show the comparison between the numerical and experimental radial distribution of the mean axial $(u)$ and tangential $(w)$ velocity profiles for the non-reacting case. These velocity profiles have been calculated at three different axial positions, $x=20,30$ and $40 \mathrm{~mm}$ downstream of the dump plane and they are normalized by the air inlet bulk velocity, $u_{0}$. The averaging-time assumed for these quantities is $\Delta T=4 \mathrm{~s}$, approximately equal to 12 times the characteristic period of the flow $(T=1 / f=1 / 3.2=0.3125 s)$ assumed as the period of PVC [21]. In general the agreement between numerical simulations and experimental data is good with the velocity peaks well predicted both in magnitude and location. The shape, size and intensity of the recirculation zone (region of negative axial velocities at the center) are well predicted, too. In fact, the numerical profiles are completely contained inside the RMS bars of the experimental data retrieved from [21].

Figure 6(a) shows the distribution of the mean axial velocity, $u$, in the burner's longitudinal plane with the zero-axial velocity isolines to highlight the recirculation zones, which are formed in the flow fluid dynamics. In particular, three distinct recirculation zones can 


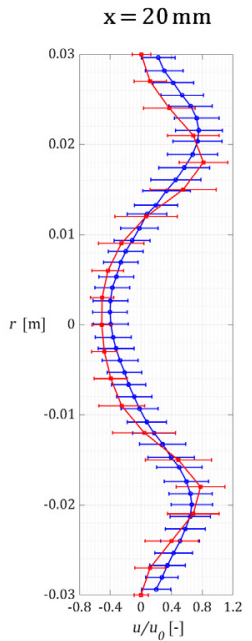

(a)

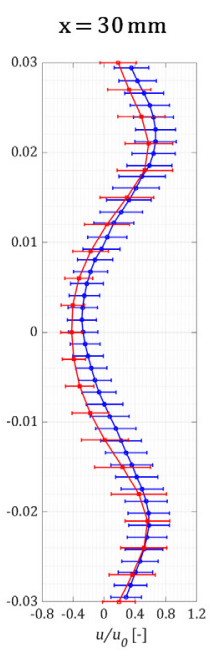

(b)

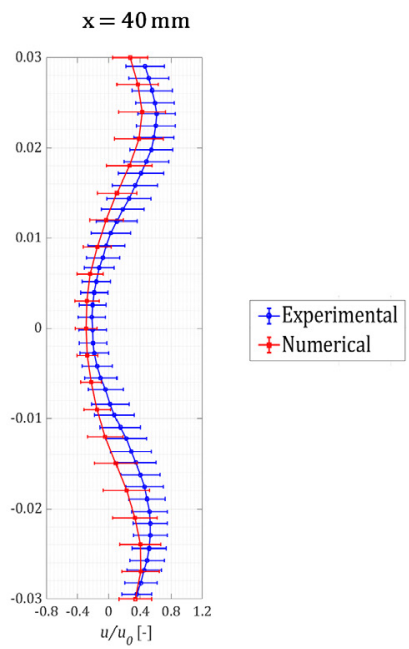

(c)

Figure 4. Comparison between numerical and experimental normalized mean axial velocity profiles, $u / u_{0}$ for non-reacting flow, at different axial locations ( $x=20 \mathrm{~mm}$ (a), $x=30 \mathrm{~mm}$ (b), $x=40 \mathrm{~mm}(\mathrm{c}))$

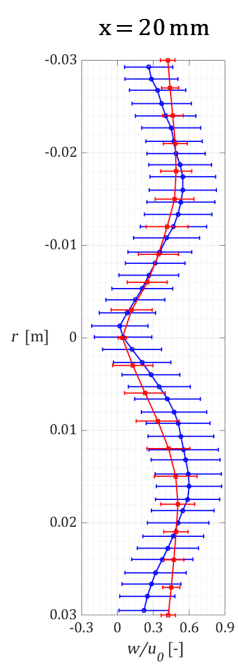

(a)

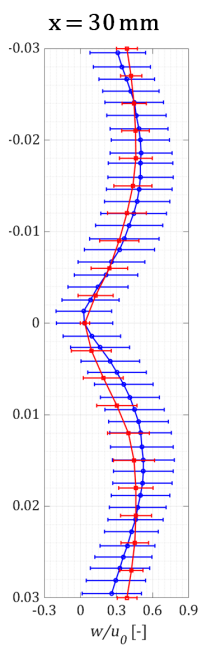

(b)

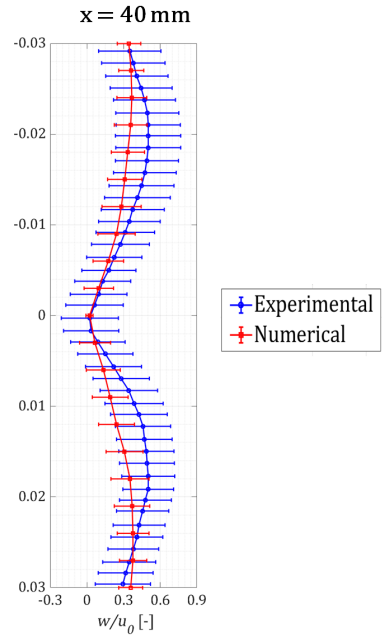

(c)

Figure 5. Comparison between numerical and experimental normalized tangential mean velocity profiles, $w / u_{0}$ for non-reacting flow, at different axial locations ( $x=20 \mathrm{~mm}$ (a), $x=30 \mathrm{~mm}(\mathrm{~b}), x=40 \mathrm{~mm}(\mathrm{c}))$

be observed: a separation Wake Recirculation Zone (WRZ) behind the center body, a Corner Recirculation Zone (CRZ) due to sudden expansion of combustor's cross section downstream of the dump plane and a Central Toroidal Recirculation Zone (CTRZ), formed due to vortex breakdown. The structure of this vortex is pointed out by the vorticity magnitude contour in logarithmic scale in Figure 6(b) where the highest vorticity levels are observed at the WRZ 
and the CTRZ. Figure 7 represents the Power Spectral Density (PSD) for the axial velocity signals sampled at the monitoring points located both on the combustor's longitudinal axis and on the traverses downstream the dump plane. It can be observed that the characteristic frequency is equal to $3.2 \mathrm{~Hz}$, as expected [21]. This one can be considered the fundamental frequency of PVC precessing motion along the axis for the non-reacting flow.

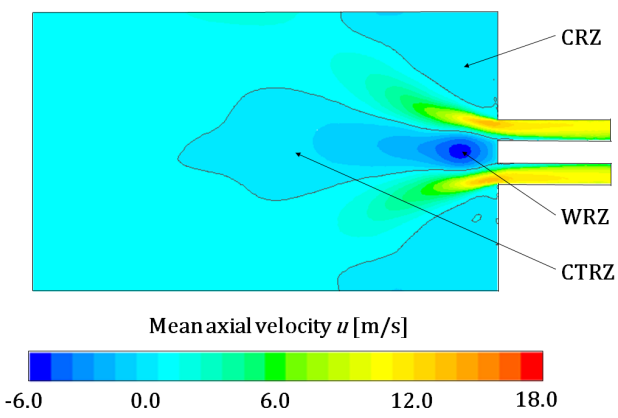

(a)

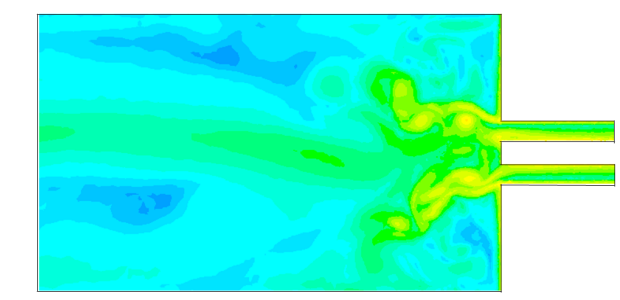

Vorticity Magnitude $\omega\left[\mathrm{s}^{-1}\right]$

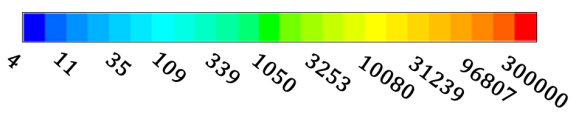

(b)

Figure 6. (a) Mean axial velocity, $u$, with identification of Wake Recirculation Zone (WRZ), Corner Recirculation Zone (CRZ) and Toroidal Recirculation Zone (CTRZ) and the zeroaxial velocity isolines in the longitudinal plane of the burner; (b) Vorticity magnitude contours.

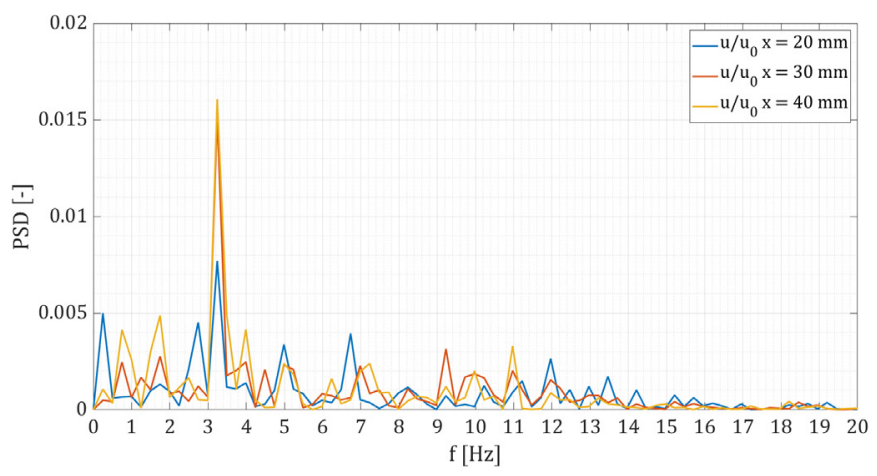

Figure 7. Power Spectral Density (PSD) for the axial velocity signals at different axial locations.

\subsection{Reacting Flow}

First of all, the mixing processes between air and the two fuel mixtures (only $\mathrm{CH}_{4}$ and $70 \% v \mathrm{CH}_{4}+30 \% v \mathrm{H}_{2}$ ) has been studied by focusing on the radial distribution of the equivalence ratio, $\phi$, entering the combustion chamber. Figure 8 shows the mean contours of the equivalence ratio, averaged on a time interval of $3 s$, at the burner's cross section located at $1 \mathrm{~mm}$ upstream the dump plane. It is noteworthy to note how $\phi$ is not homogeneous $(\phi=0.7)$. Moreover, its gradient in the radial direction in the case of methane-hydrogen mixture injection is higher than the case of pure methane injection: the fuel, indeed, tends to concentrate at 
the outermost radii. This is a typical issue encountered in adapting combustors to hydrogen enriched fuels: because of the density reduction of fuel mixture due to the hydrogen blending, its momentum $\left(\rho u_{r}^{2}\right)$ increases in the injection, ensuring a greater penetration in the main air flow and letting the mixture leaner at inner radii.

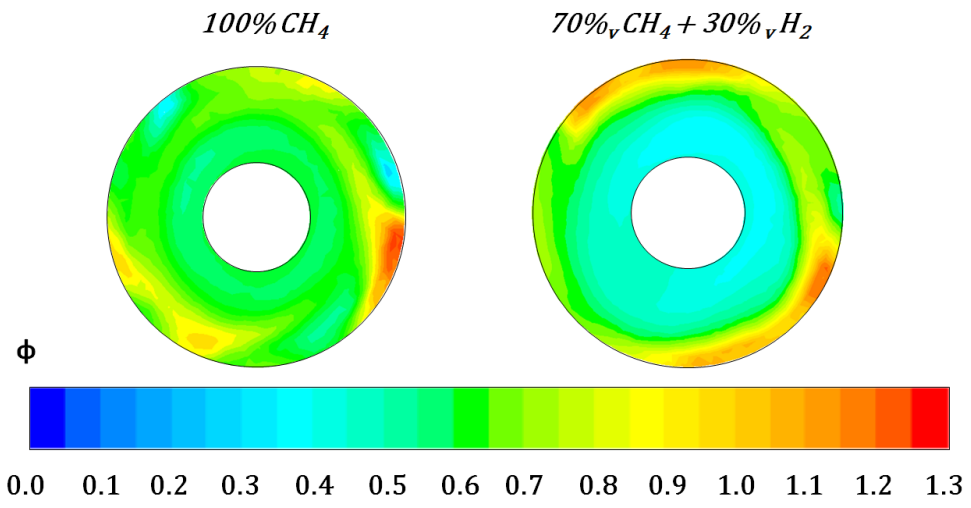

Figure 8. Comparison of the mean contours of the equivalence ratio, $\phi$, between pure methane (left) and methane-hydrogen mixture (right) combustion case (evaluated at the cross section located at $1 \mathrm{~mm}$ upstream of the dump plane.

Figure 9 shows the comparison of axial velocity distribution, $u$, into the burner's longitudinal plane between pure methane (a) and methane-hydrogen mixture (b) combustion case. The iso-velocity lines for $u=0 \mathrm{~m} / \mathrm{s}$ have been drawn to point out the recirculation zones. What is immediately marked is the similarity between the two velocity fields. this confirms the fact that up to $30 \%$ the velocity field of motion does not change significantly. Moreover, if we compare this field with the one of the non-reacting case (see Figure 6b), we can notice a variation of CTRZ in dimension and intensity. Indeed, it tends to stretch towards the outlet and to increase its radial dimension, leading the flow towards the lateral walls of the combustion chamber. This behaviour has been found in other works on burners with a geometry similar to that investigated in this work (see for example [23]).

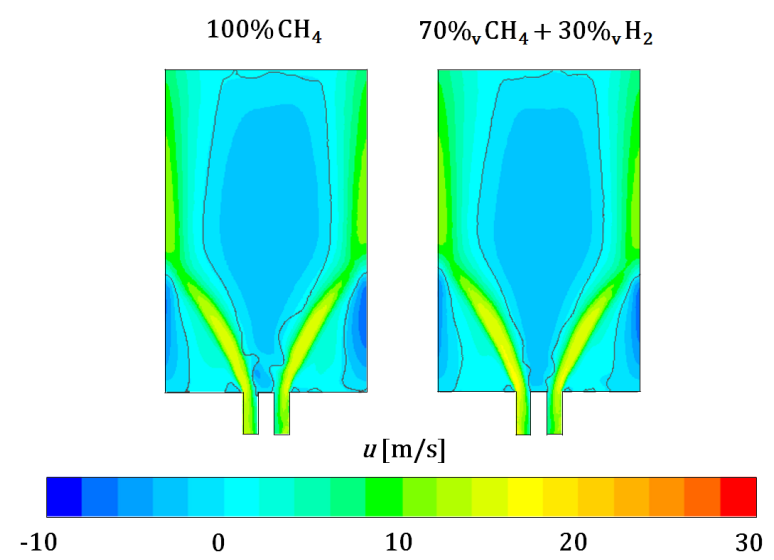

Figure 9. Comparison of axial velocity distribution, $u$, into the longitudinal plane of the burner between pure methane (left) and methane-hydrogen mixture (right) combustion case. 
Table 2: $\mathrm{CO}_{2}$ emissions computed for both pure methane and methane-hydrogen mixture cases

\begin{tabular}{cccc}
\hline & $\begin{array}{c}\text { Mean Mass Fraction } \\
{\left[\mathrm{kg}_{\mathrm{CO}_{2}} / \mathrm{kg}_{\text {mix }}\right]}\end{array}$ & $\begin{array}{c}\text { Mean Molar Fraction } \\
{\left[\mathrm{kmol}_{\mathrm{CO}_{2}} / \mathrm{kmol}_{\text {mix }}\right]}\end{array}$ & $\begin{array}{c}\text { Concentration } \\
{\left[\mathrm{gCO}_{2} / \mathrm{Nm}_{\text {mix }}^{3}\right]}\end{array}$ \\
\hline $100 \% \mathrm{CH}_{4}$ & 0.106 & 0.0675 & 132.6 \\
$70 \% \mathrm{v} \mathrm{CH}+30 \% \mathrm{H}_{2}$ & 0.0964 & 0.0607 & 119.2 \\
\hline
\end{tabular}

The contours of mean static temperature field represented into the combustion chamber for the two reacting flows are compared in Figure 10. A higher temperature can be noted in the reaction zone for the case of methane-hydrogen mixture (2260 K for the mixture of methane enriched with $30 \% v$ of hydrogen versus $2150 \mathrm{~K}$ for the pure methane). This is due to two factors: firstly the introduction of hydrogen into the fuel mixture because of its slightly greater adiabatic flame temperature than methane; the second point is the higher local equivalence ratio of the mixture at the outer diameter of the mixing tube.

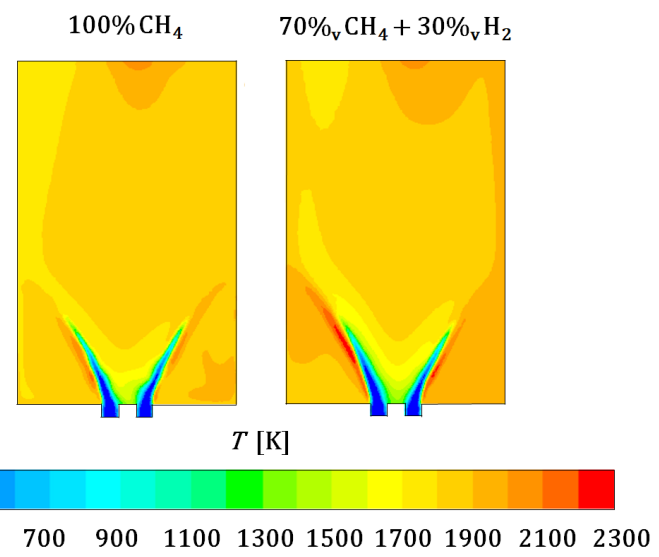

Figure 10. Comparison of mean static temperature field into the longitudinal plane of the burner between pure methane (left) and methane-hydrogen mixture (right) combustion case.

Instead, in Figure 11 and Figure 12 the radical $\mathrm{OH}$ and the $\mathrm{CO}_{2}$ mass fraction distributions are represented for both cases, respectively. The first ones allow to highlight the reaction zone into the combustion chamber because they are intermediate radicals of the combustion mechanism. The higher production of $\mathrm{OH}$ in methane-hydrogen mixture's combustion points out the greater chemical reactivity of the hydrogen enriched mixture than pure methane, especially in the outer recirculation zone. Regarding to $\mathrm{CO}_{2}$ production, they result lower for the combustion of methane-hydrogen mixture because of the lower carbon content in comparison to pure methane. In the Table 2 the $\mathrm{CO}_{2}$ emission levels are shown and it has been estimated a $10 \%$ reduction for the hydrogen enriched mixture. However, there is still a certain degree of asymmetry into the mean contours of the different quantities of the reacting flows, which could be caused by a short average time interval due to the high computational cost. 


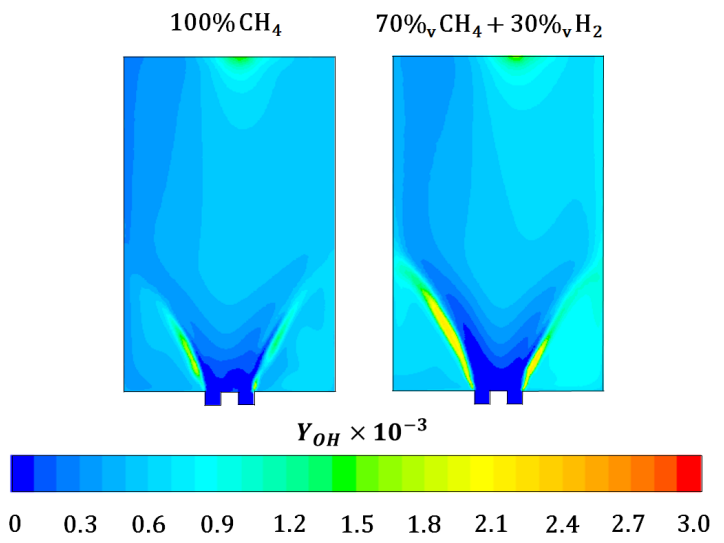

Figure 11. Comparison of $\mathrm{OH}$ mass fraction distribution into the longitudinal plane of the burner between pure methane (left) and methane-hydrogen mixture (right) combustion case.

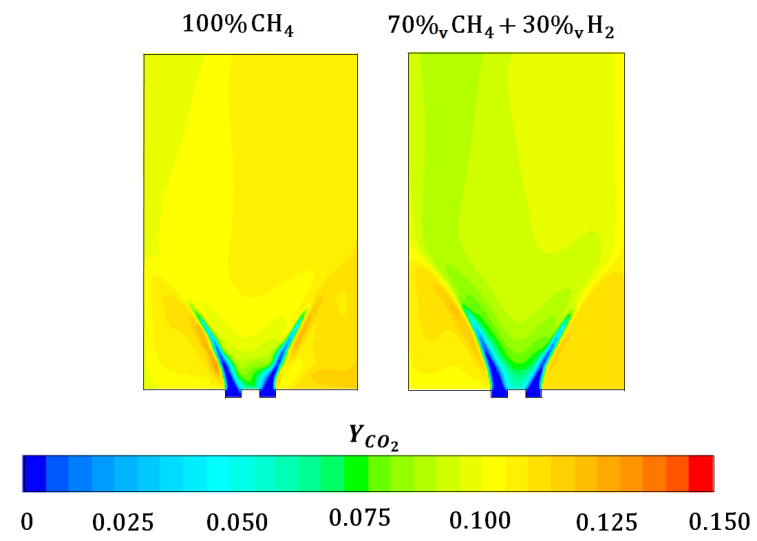

Figure 12. Comparison of $\mathrm{CO}_{2}$ mass fraction distribution into the longitudinal plane of the burner between pure methane (left) and methane-hydrogen mixture (right) combustion case.

\section{Conclusions}

In conclusion, in this work the main advantages and disadvantages related to combustion with hydrogen are highlighted and the thermo-fluid dynamic study conducted on a swirl-stabilized lean-premixed laboratory-scale burner is presented. This burner, both in the case of cold and hot flow, has been simulated by means of the commercial software ANSYS Fluent ${ }^{\circledR}$. In the numerical analysis of the non-reacting flow a good agreement with the experimental velocity fields into the burner has been reached and it has been possible to highlight the structure of the PVC characterized by a precessing motion with a frequency of $3 \mathrm{~Hz}$. Then, an analysis of mixing between the oxidant (air) and the injected fuel has been carried out in order to underline the effect due to the addition of $30 \% v$ of hydrogen. This study has pointed out a difference in the mean distribution of the equivalence ratio by introducing hydrogen in the fuel mixture: because of the density reduction of fuel mixture due to the hydrogen blending, the momentum of the fuel flow increases in the injection, ensuring a greater penetration in the main oxidizing air flow and letting the mixture leaner at inner radii. For this reason, the partially premixed combustion model has been implemented to take into account these 
inhomogeneities of the mixture at the chamber inlet. Finally, referring to the reacting flows, it has been shown a variation of CTRZ in dimension and intensity with respect the nonreacting case, with a significant stretching towards the outlet and an increment in the radial direction, as well as a reduction approximately equal to $10 \% v$ of carbon dioxide production. In the future, further investigations will be carried out in order to focus on $N O_{x}$ production.

\section{References}

[1] N. Z. Muradov, T. N. Veziroğlu, International journal of hydrogen energy 30 (3) (2005).

[2] G. Hu, C. Chen, H. T. Lu, Y. Wu, C. Liu, L. Tao, Y. Men, G. He, K. G. Li, Engineering 6 (12) (2020), pp. 1364-1380.

[3] R. L. Edwards, C. Font-Palma, G. Howe, Sustainable Energy Technologies and Assessments 43 (2021), 100901.

[4] A. Kovač, M. Paranos, D. Marciuš, International Journal of Hydrogen Energy 46 (16) (2021), pp. 10016-10035.

[5] W. Liu, F. Wen, Y. Xue, Journal of Modern Power Systems and Clean Energy 5 (3) (2017), pp. 439-450.

[6] P. Chiesa, G. Lozza, L. Mazzocchi, J. Eng. Gas Turbines Power 127 (1) (2005), pp. 73-80.

[7] T. G. Reichel, "Flashback prevention in lean-premixed hydrogen combustion" (Doctoral dissertation, Technische Universität Berlin) (2017).

[8] T. G. Reichel, S. Terhaar, C. O. Paschereit, Journal of Propulsion and Power 34 (3) (2018), pp. 690-701.

[9] T. G. Reichel, K. Goeckeler, C. O. Paschereit, Journal of Engineering for Gas Turbines and Power 137 (11) (2015).

[10] T. Capurso, V. Ceglie, F Fornarelli, M. Torresi, S. M. Camporeale, E3S Web of Conferences 197 (2020), 10002.

[11] M. Wang, Y. Zhong, K. Deng, AIP Advances 9 (4) (2019), 045206.

[12] E. Æsøy, J. G Aguilar, S. Wiseman, M. R. Bothien, N. A. Worth, J. R. Dawson, Combustion and Flame 215 (2020), pp. 269-282.

[13] T. Indlekofer, B. Ahn, Y. H. Kwah, S. Wiseman, M. Mazur, J. R. Dawson, N. A. Worth, Combustion and Flame, 228 (2021), pp. 375-387.

[14] J. Beita, M. Talibi, S. Sadasivuni, R. Balachandran, Hydrogen 2 (1) (2021), pp. 33-57.

[15] Y. Pyo, D. Kim, S. K. Kim, D. J. Cha, Journal of Mechanical Science and Technology 33(11) (2019), pp. 5547-5559.

[16] ETN, Hydrogen Gas Turbines report - The path towards a zero-carbon gas turbine (2020).

[17] O. Tuncer, S. Acharya, J. H. Uhm, in Proceedings of ASME Power 2006, (2006).

[18] O. Tuncer, S. Acharya, J. H. Uhm, International Journal of hydrogen energy, 34 (1) (2009), pp. 496-506.

[19] A. De, S. Acharya, International journal of hydrogen energy 37 (22) (2012).

[20] O. Tuncer, "Active control of spray combustion" (PhD thesis, Louisiana State University, Louisiana) (2006).

[21] A. De, S. Zhu, S. Acharya, in Proceedings of ASME Turbo Expo 2009: Power for Land, Sea and Air, (2009), GT2009-60230.

[22] GRI-Mech Web site http://www.me.berkeley.edu/gri-mech.

[23] D. Mira, O. Lehmkuhl, A. Both, P. Stathopoulous, T. Tanneberger, T. G. Reichel, C. O. Paschereit, M. Vazquez, G. Houzeaux, Turbulence and Combustion 104 (2020), pp. 479-507. 